\title{
Shared Spatial Situation Awareness as a Team Performance Indicator in Collaborative Spatial Orientation Task
}

\author{
Baptiste Prebot ${ }^{1,2}$, Jean-Marc Salotti $^{1,3}$, Coralie Vennin ${ }^{1}$, Bernard Claverie ${ }^{1}$, \\ ${ }^{1}$ ENSC, IMS laboratory, Bordeaux INP, Bordeaux University, CNRS, 109 Avenue Roul, \\ 33400 Talence, France \\ ${ }^{2}$ DGA Talence, France \\ ${ }^{3}$ Auctus, INRIA, Talence, France \\ \{baptiste.prebot, bernard.claverie, coralie.vennin, jean-marc.salotti\} @ensc.fr
}

\begin{abstract}
The present study investigates the link between time taken by a team to perform a spatial orientation task and the evaluation of spatial shared situational awareness (SSA). Paired in teams, volunteers have to collaborate to send a vehicle to a specific location on a computer simulation as quickly as they can. The roles and information they have to reach that goal are different. Every 45 seconds participants are asked to mark on their map the location they believe the vehicle to be. Along with its real position, these marks are used to objectively evaluate spatial SSA. First results allow us to divide participants into three groups in accordance with Endsley's distinction of Shared SA evaluation. Interestingly, fastest teams were not the ones with the most accurate and shared spatial representation of the situation. Potential use of such indicators in team training is outlined.
\end{abstract}

Keywords: Collaboration - Shared Situation Awareness - Spatial Awareness · Team Performance

\section{Introduction}

We address the problem of team shared situation awareness assessment in complex organizations with complex decision making processes. Applications can be found for examples in the context of command and control in the military domain or in the context of scientific exploration on far planetary surfaces. The assessment of shared situation awareness (SSA) is usually carried out a posteriori (e.g., after an accident) and in general by means of a qualitative analysis. An important difficulty is to be able to make a quantitative measurement of variables that are correlated to the exactness of the situation awareness and to the similarity of the shared representation. It is proposed here to analyze the spatial component of situation awareness, which can be directly inferred from the marks that participants can put on a map to indicate a belief of position. Depending on the quality of the collaboration, the marks can be more or less exact and similar for all participants of the same team. An experiment has been carried out in a virtual environment to test the SSA and to examine the links between the spatial 
SSA and task performance. The paper is organized as follows. Section 2, the method is presented and the experiment is described. Section 3, the main results are given and discussed. In conclusion, some perspectives are proposed.

\section{Method}

\subsection{Main Concepts}

Collaboration is seen in this study as the integrative form of cooperation, as defined by Schmidt [1], where agents are engaged in subtasks of the same main task, while having different but complementary skills. This idea of complementarity and role division under a common goal is also found in Salas, Dickinson, Converse and Tannenbaum [2] definition of a team: "a distinguishable set of two or more people who interact dynamically, interdependently, and adaptively toward a common and valued goal/object/mission, who have each been assigned specific roles or functions to perform, and who have a limited life span of membership" (p. 126-127).

From search and rescue missions to space exploration or military operations, the experimental conditions of two separated operators, one having direct impact on the environment and the other having more information, is a common occurrence. In such collaborative environments, the shared ability to guide one another and localize objects in unknown terrains, in real time and possibly in degraded situation is critical for the overall team performance. To do so efficiently, teammates must share the most common and accurate representation of the situation as possible.

Already a widely studied subject in the field of human factors, situation awareness (SA) has gained even more interest in the rise of team cognition and performance evaluation. Since the 1980s numerous models have been proposed [3][4]. Endsley formally defines it as "...the perception of the elements in the environment within a volume of time and space, the comprehension of their meaning, and the projection of their status in near future" (p. 36)[5]

Extending the concept to teams and collaborative work Shared SA can be defined as "the degree to which team members possess the same SA on shared SA requirements" (p.48)[6]. As Shared SA can be seen as a matter of both knowledge and coordination, two levels of measurement are distinguished [7]. The degree of accuracy of an individual's SA and the similarity of two individual's SA are both needed in order to assess the Shared SA between two team members. The evaluation of SA accuracy is what most evaluation techniques are focused on. For each participant of the observed experiment, the understanding of the situation is compared to the true state of the environment at the time of evaluation, leading to the assessment of SA as being either right or wrong. This binary evaluation requires a ground truth to which the SA has to be compared. The evaluation of the similarity of SAs is based on the direct comparison of situation awareness elements, which are relevant to both of them. The assessed SA is either shared or not on these specific elements. Combining evaluations of each participant lead to SA being assessed as either right or wrong and shared or not, defining three possible SSA states: different, both correct, both incorrect [6]. 
When guiding someone remotely, the current position of the person being guided in the field can be defined as a necessary shared knowledge element [8], a must-shared information for the correct accomplishment of the collaborative task.

In this study the focus is put on a spatial orientation task [9]. Thus, working on the sharing of spatial representation of the situation, we consider here spatial situation awareness [10] as a part of SA, a restriction of the one's global understanding of the situation to only some elements relative to the position in space of the teammate or of oneself.

The overall goal of this study is to investigate how spatial Shared SA similarity and accuracy relate to the team performance (here the time spent to accomplish the task) and in what extent they can themself be used as objective quantitative indicators of team's performance.

The research questions can be summarized as follow:

- Can Spatial SSA Accuracy and Similarity be used as quantitative team performance metrics?

- Can Spatial SSA Accuracy and Similarity be used to identify teams profile in regard of their performance to the collaborative task?

\subsection{Description of the Experiment}

62 participants (38 female and 24 male) ranging from 18 to 43 years old $(M=21.6)$ took part in this study. They were recruited around the local campus to perform a collaborative orientation task. Paired up in teams, volunteers have to collaborate to send a vehicle (a rover) to a specific location on a computer simulation of a Martian environment as quickly as they can. To do so, they are assigned two specific roles. One person, the astronaut (Astro), drives the rover in the virtual environment and has a map of that environment, while the second person, the captain (Capcom) in charge of guiding him, only has access to the map, with the target (a white rock) location marked. They had no previous knowledge of the environment and were separated so as to be able to communicate only orally.

\subsection{Material}

A A4-size paper map representing the simulated environment was given to each participant. No scale was indicated nor any grid or coordinate system. Only the starting point of the rover and its orientation were stipulated on each map. Maps were given oriented in the same way to each participant. The simulation is Unity based, developed internally, and was displayed on a 24-inch monitor. 


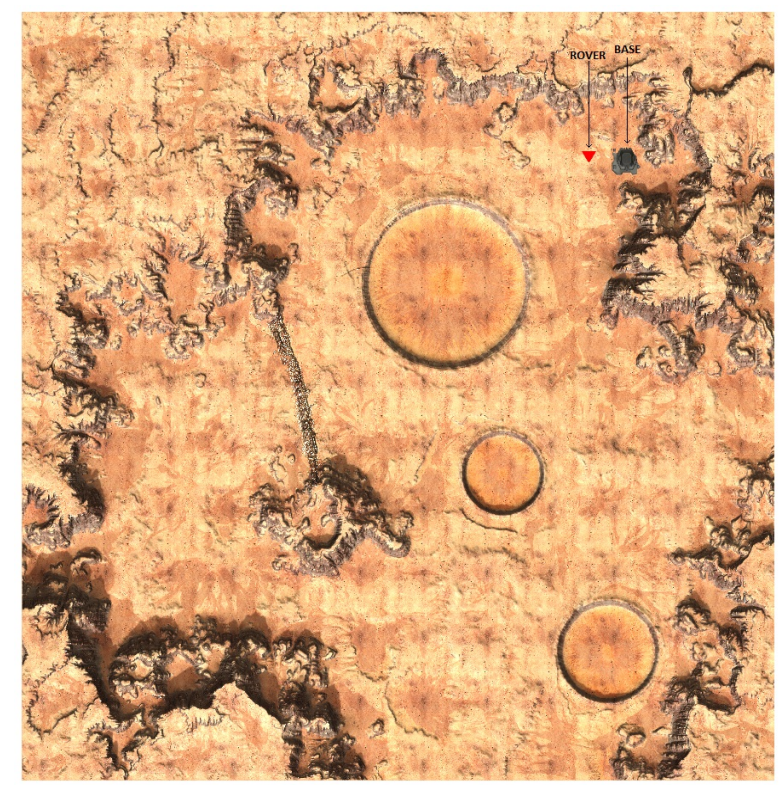

Fig. 1. Example of the map given to the Astro

\subsection{Design and Procedure}

Before the experiment, each teammate was assigned the role either of Astro or Capcom. Then they were brought to their work station and given instructions depending on their role. The map was handed to them and the starting position of the rover and initial orientation was indicated on it. The Astro task was to navigate the rover (first person view of the environment, as he was the driver), following directions given by CapCom, in order to find a specific rock. They were told that the goal was to find the rock as quickly as possible. During the whole experiment they were allowed to communicate only orally. CapCom was instructed not to directly give the Astro the rock position (not allowed to say "the rock is on the top left corner of the map") but to orient him in realtime. Every 45 seconds the simulation was paused and the subjects were asked to mark down on their map where they estimate the rover to be (Position Evaluation Point). Teammates were not allowed to communicate during this phase of the experiment. If after 15 minutes, participants did not find the rock, it was considered a failure and the simulation was stopped. After the experiment, they answered a questionnaire composed of background questions and subjective ratings and feedbacks on the evaluation of their collaboration. 


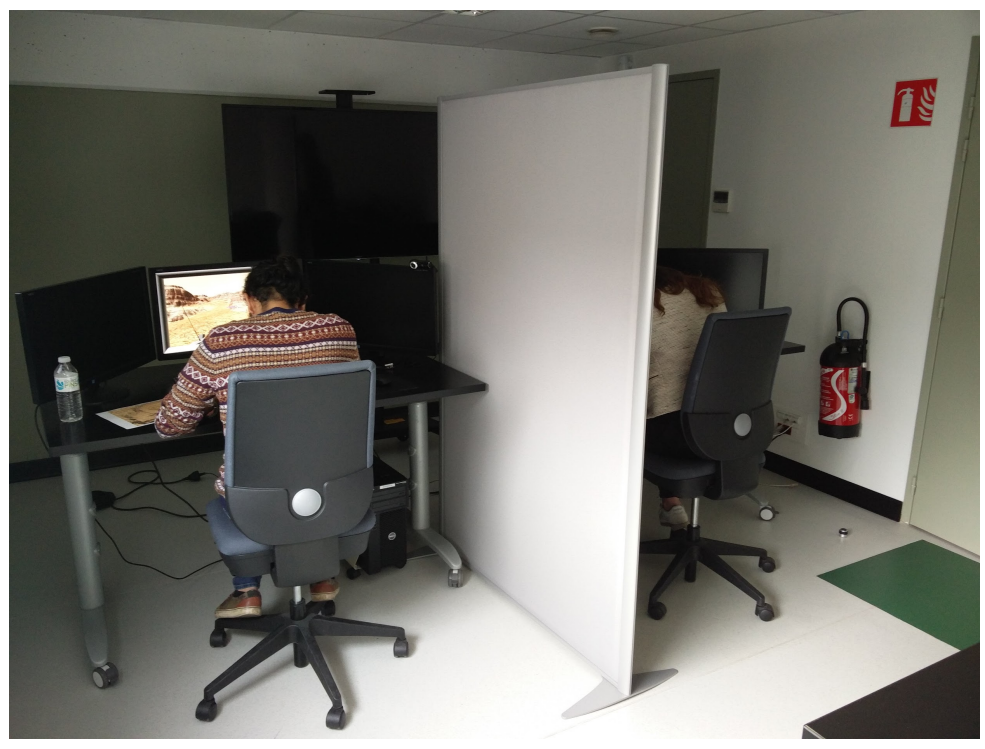

Fig. 2. Experimental set-up

\subsection{Measures}

Three types of quantitative team performance metrics have been recorded. Two spatial metrics are extrapolated from the marked positions on the map. Spatial SSA accuracy is objectively measured by comparing the position of the marks on each map with the exact position that is registered by the simulation. By measuring the distance between the positions marked by teammates for a same position evaluation point we evaluated the similarity of spatial SSA. The distance is expressed in Unity measurement metric (1 unit $=0.083 \%$ of the map). Finally, the time taken (in seconds) to complete the task has been recorded and serves as the Time performance metric.

Using Likert scales, participants' subjective evaluation of the team performance, their own and their teammate performance, their quality of sharing of SA and the perceived difficulty of the task have been collected through a post-experiment questionnaire.

\section{$3 \quad$ Results}

Among the 31 tested pairs, 3 crashed the vehicle. These 3 experiments have not been considered for situation awareness assessment. Among the remaining 28 pairs, 8 did not find the rock under 15 minutes. As they failed to complete the task, they have been grouped in a separate category. Though it is interesting to look at the cause of failure, we propose to focus in this paper only on the 20 successful teams. The mean time to complete the task is $460.5 \mathrm{~s}(\min =274 \mathrm{~s} ; \max =648 \mathrm{~s}, \mathrm{SD}=102.56)$. SSA similarity and SSA accuracy have been calculated. The results are presented and analyzed in the next paragraphs. 


\subsection{SSA Similarity}

The 42 participants generated 386 Position Evaluation Points. Coordinates of each point have been logged. Each Astro point was paired with the corresponding Capcom point. For each pair, the distance between the two points is calculated. The shorter the distance between the two points, the more similar, and thus shared, the representation of the spatial position is between the teammates. Then for each team, distances have been averaged across the number of points that have been marked on their maps.

\subsection{SSA Accuracy}

As stated earlier, the real position of the rover has been registered by the simulation all along the experiment. Each Position Evaluation Point coordinates have been compared to the real position of the rover recorded by the simulation at that time.

The distance between the two points is calculated. Averaging the distances by subjects shows a difference between Astro and CapCom SSA accuracy. Astros mean SA accuracy is lower (Mean Astro=97.67, $\mathrm{SD}=107.02$ ) than CapComs mean accuracy (Mean CapCom=141.06, SD=137.03), meaning that Astros have generally a more accurate representation of their spatial location than CapComs. This is coherent since Astro is the one whose position is evaluated and the one navigating the simulation, thus more inclined to have a more accurate evaluation of his own position in the environment. For a team level evaluation, the accuracy distances for each point and each teammate have been averaged. We obtain a single average accuracy value for each team. As for SSA Similarity, the lower the mean value of the distance between those points is, the more accurate the SA is. Perfect accuracy would be 0 . Note that the more accurate the team is, the more similar it is supposed to be.

Table 1. Statistics of SSA Similarity, Accuracy and Time measurements.

\begin{tabular}{llll}
\hline & Similarity & Accuracy & Time (s) \\
\hline Mean value & 94,87 & 103,62 & 457 \\
Standard deviation & 71,40 & 65,28 & 104.14 \\
Min & 4,54 & 46,16 & 274 \\
Max & 339,52 & 318,87 & 648 \\
\hline
\end{tabular}

\subsection{Group Analysis}

As seen in Figure 3, two teams can clearly be identified from their Shared SA Accuracy and Similarity performance. Team $\mathrm{N}^{\circ} 16$ (G16, top right corner of the plot) possess mean Similarity and Accuracy scores both more than two times over the average values $($ Similarity $=201.08$, Accuracy $=238.17)$. Both teammates are being incorrect in different ways, thus having an inaccurate and really different representation of the situation. Team $\mathrm{N}^{\circ} 10$ (G10, bottom right) is the less accurate one, with a mean Accuracy of 318.87 , more than three times the average value, while interestingly the mean Similarity is under the average value (81.39). It reflects teammates sharing a common but erroneous representation of the situation. 
Surprisingly, Time performance of these two teams is average. A further analysis of their collaboration would be required.

These two specific cases cover two of the three possible Shared SA states as described by Endsley [6], with inaccurate and either similar or not representation of the situation.

The following analysis is focusing on the remaining 18 teams as having an overall accurate and similar SSA but showing a great variability in Time performance.

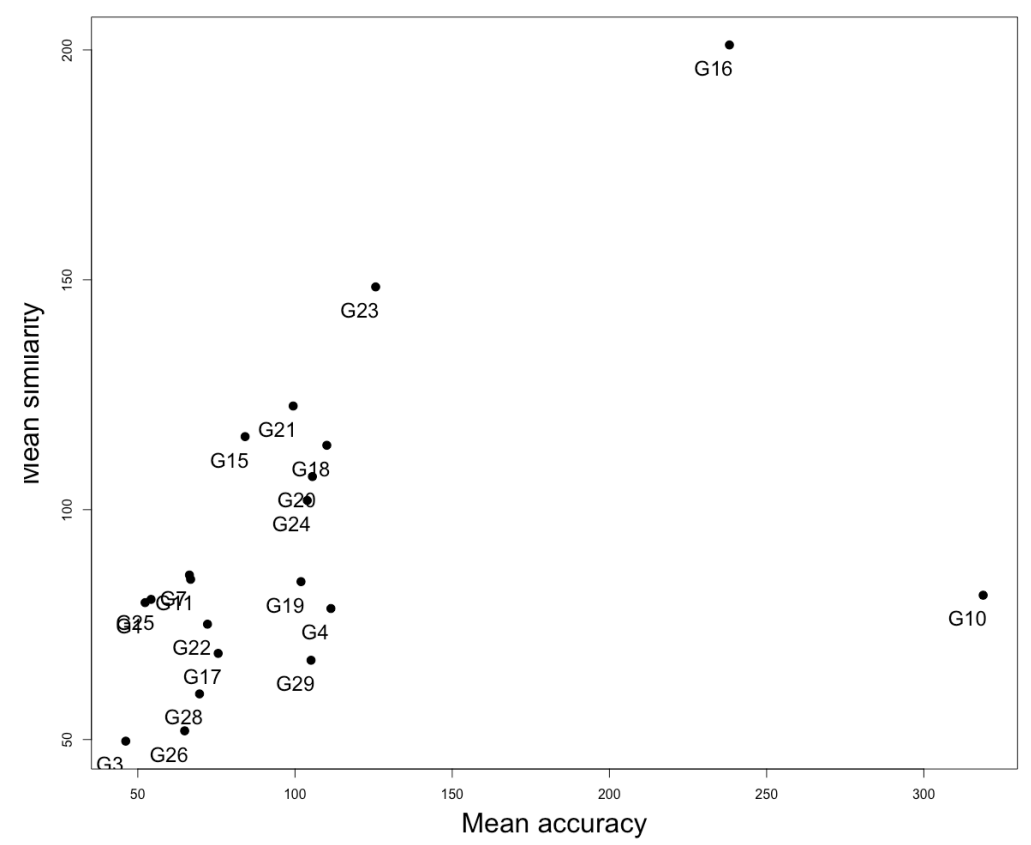

Fig. 3. Teams plotted by Similarity and Accuracy SSA performance

\subsection{K-means Analysis}

In order to find possible clusters of teams based on their SSA accuracy, SSA similarity and Time performance, a K-means clustering method was used, implemented in R package of Morisette and Charier [11], based on Hartigan and Wong algorithm [12]. The algorithm classifies teams according to the similarity criterion and determines for each class a referent vector. For each class, the sum of the square distances between each team vector and the referent vector represents the inter-individual variability. Then, the sum of the square distances between each pair of referent vectors describes the variability between each class. The number of teams assigned to each class is also defined.

From the K-means analysis, 2 groups can be characterized, both composed of 9 teams. Descriptive statistics of each identified cluster are presented in Table 2. 
Table 2. Statistical features of the two clusters of teams obtained with K-Means.

\begin{tabular}{lll}
\hline & Cluster 1 & Cluster 2 \\
\hline Mean Similarity & $63.1(13.8)$ & $105.2(24,9)$ \\
Mean Accuracy & $70.6(9.9)$ & $104.4(11)$ \\
Mean Time & $381.8(77.2)$ & $545.4(57)$ \\
Sum of Squares & 49964.9 & 31936.5 \\
min(Mean Accuracy) & 46.1 & 84.1 \\
$\max ($ Mean Accuracy) & 75.5 & 125.6 \\
$\min ($ Mean Similarity) & 49.6 & 67.2 \\
$\max ($ Mean Similarity) & 85.7 & 148.4 \\
$\min ($ Mean Time) & 274 & 476 \\
$\max ($ Mean Time) & 462 & 648 \\
$\mathrm{~N}$ & 9 & 9 \\
\hline
\end{tabular}

Results show that the first cluster identified has a better overall Shared SA performance. Teams in Cluster 1 have position evaluations that are closer to the reality. The average calculated for the accuracy variable is equal to $70.6(\mathrm{SD}=9.9)$ while it is equal to $104.9(\mathrm{SD}=11)$ for the second cluster. The standard deviations for the Accuracy and Similarity variables indicate that the evaluation performance is more consistent in cluster 1 than in cluster 2 . The results for the time variable show that the average duration of the task is better in the first cluster. The teams took on average 381 seconds $(\mathrm{SD}=77.2)$ to find the rock whereas the second cluster took on average 545 seconds $(\mathrm{SD}=57)$. Standard deviations also indicate that cluster 1 has a bigger interteams variability. Indeed, the minimum duration is 274 seconds and the maximum is 462 seconds. In the second cluster the minimum duration is 476 seconds when the maximum duration is 648 seconds. As can be seen from the boxplots of Figure 1, the confidence intervals measured for the Time variable indicate that the temporal performances are significantly different between the clusters 1 and 2 . The same can be said for the SA accuracy. 

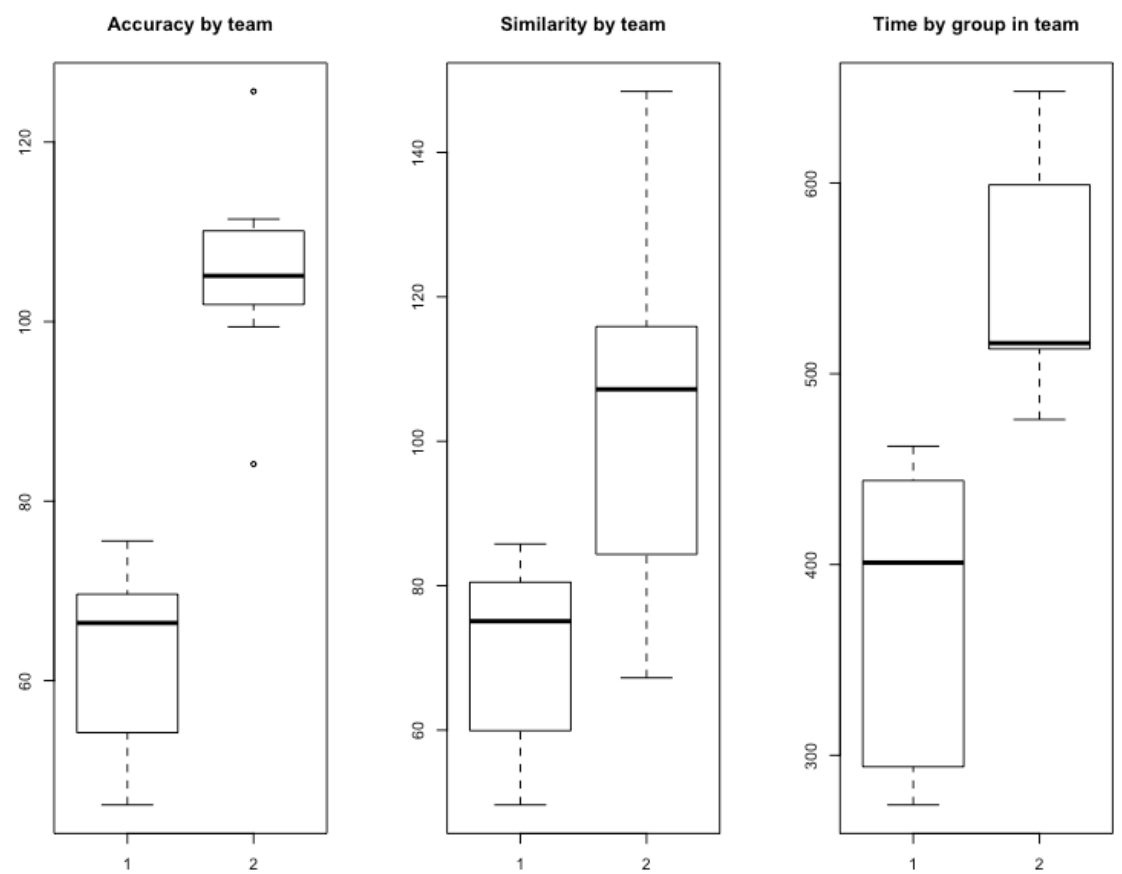

Fig.4. Boxplot comparison of SSA Accuracy, Similarity and Time performances by team.

Based on this observation, a relationship can be assumed between mean SA accuracy and Time performance. Pearson's correlation test allowed us to verify this relationship. The result confirm a positive linear relation between Shared SA Accuracy and Time performance $(\mathrm{r}=0.76 ; \mathrm{p}<.001)$. The smaller the mean SSA Accuracy distance is over the experiment, the smaller, thus better the time performance will be.

\section{Discussion}

Several results have be presented regarding the link between spatial Shared Situation awareness evaluation and time performance during a collaborative orientation task. First, the two specific cases of "inaccurate and different", and "inaccurate but similar" Shared SA have been identified.

When focusing our analysis on the task completed teams in terms of Shared SA, two groups emerged with a correlation between SSA and time performance. Although the overall spatial SSA difference alone is not enough to explain the inter-teams differences in Time performance, results reveal that spatial SSA Accuracy is positively correlated to it. This correlation implies that spatial SSA Accuracy may be used as a quantitative linear indicator to anticipate team's time performance.

However, Spatial SSA Accuracy might not be the only variable to take into account in order to explain Time performance. Previous work [13] already examined the use of common experience, workload similarity or communication distances, as predictors of 
shared situation awareness in teams. Complementary, integrating in our model answers from the post-experiment questionnaire could help identify if other factors, like individual perceived task difficulty and perceived team or teammate's performance, can be used to predict their performance. This will probably be the center of future work.

In sum, using distances (spatiality) as an evaluation metric of Shared SA, allowed us to quantify more precisely the situation awareness, giving levels to in the end qualify teams performance.

The findings presented here provide preliminary evidence that it is possible, in a collaborative spatial orientation task, to profile teams and anticipate, to some extent, their expected performance. Finally, in the domain of astronautics, this method can be used for training and testing astronauts' behavioral competency, especially situation awareness optimization and communication efficiency, which are in the list of competencies required by NASA [14]. Other applications could include defining an optimal performance matching the team collaboration profile or tailoring training simulations and exercises goals to each team.

Future work will also include analysis of the intra-teams temporal evolution of Spatial SSA metrics during the experiment.

Acknowledgments. Part of this study has been financed by the Direction Générale de l'Armement (DGA). We would like to particularly thank Laetitia Calice, Caroline Cavel, Mateo Mahaut and Adrien Leduque, ENSC students who have been highly involved in the experiment.

\section{References}

1. K. Schmidt, "Cooperative work: A conceptual framework," Distrib. Decis. Mak. Cogn. Model.Coop. Work, vol. 21,pp. 75-110, 1991.

2. S. I. Salas, E.; Dickinson, T.L.; Converse, S.A.; Tannenbaum, "Toward an understanding of team performance and training.," Teams Their Train. Performance;, vol. Volume 12, p. 329., 1992.

3. N. A. Stanton, P. M. Salmon, G. H. Walker, E. Salas, and P. A. Hancock, "State-of-science: situation awareness in individuals, teams and systems," Ergonomics, vol. 60, no.4,pp. 449466, 2017.

4. P. M. Salmon, N. a. Stanton, G. H. Walker, and D. P. Jenkins, "What really is going on? Review of situation awareness models for individuals and teams," Theor. Issues Ergon. Sci., vol. 9, no. 4, pp. 297-323, 2008.

5. M. R. Endsley, "Toward a Theory of Situation Awareness in Dynamic Systems," Hum. Factors J. Hum. Factors Ergon. Soc., vol. 37, no. 1, pp. 32-64, Mar. 1995.

6. W. M. Endsley, Mica and Jones, "A model of inter and intra team situation awareness: Implications for design, training and measurement. New trends in cooperative activities: Understanding system dynamics in complex environments," New trends Coop. Act. Underst. Syst. dyanmics complex Environ. (Santa Monica, CA Hum. FActors Ergon. Soc., pp. 46-67, 2001.

7. L. D. Saner, C. A. Bolstad, C. Gonzalez, and H. M. Cuevas, "Measuring and Predicting Shared Situation Awareness in Teams," J. Cogn. Eng. Decis. Mak., vol. 3, no. 3, pp. 280$308,2009$. 
8. C. D. Wickens, "Situation awareness: review of Mica Endsley's 1995 articles on situation awareness theory and measurement.," Hum. Factors, vol. 50, no. 3, pp. 397-403, 2008.

9. B. J. E. Johansson, C. Hellgren, P.-A. Oskarsson, and J. Svensson, "Supporting situation awareness on the move-the role of technology for spatial orientation in the field," Proc. 10th Int. ISCRAM Conf. - Baden-Baden, Ger. May 2013, no. May, pp. 442-451, 2013.

10. A. Klippel, S. Hirtle, and C. Davies, "You-are-here maps: Creating spatial awareness through map-like representations," Spat. Cogn. Comput., vol. 10, no. 2-3, pp. 83-93, 2010.

11. L. Morissette and S. Chartier, "The k-means clustering technique: General considerations and implementation in Mathematica," Tutor.Quant. Methods Psychol., vol. 9, no. 1, pp. 1524, 2016.

12. J. A. Hartigan and M. A. Wong, "Algorithm AS 136: A K-Means Clustering Algorithm," Appl.Stat., 2006.

13. C. A. Bolstad, P. Foltz, M. Franzke, H. M. Cuevas, M. Rosenstein, and A. M. Costello, "Predicting Situation Awareness from Team Communications Pearson Knowledge Technologies," pp. 789-793, 2007.

14. L. Bessone et al., "International Space Station Human Behavior \& Performance Competency Model Volume II Mission Operations Directorate,” vol. 2, no. April, 2008. 